\title{
Caractéristiques et dynamique des mauvaises herbes en région de grande culture : le Noyonnais (Oise)
}

Jean-Pierre LONCHAMP et Gilbert BARRALIS

I.N.R.A., Laboratoire de Malherbologie, B.V. 1540, F 21034, Dijon Cedex

RÉSUMÉ

\begin{abstract}
Après avoir rappelé l'importance prépondérante du désherbage et du rôle des herbicides en régions de grande culture, les auteurs se proposent d'étudier l'efficacité des moyens de lutte utilisés et leur incidence sur la densité d'infestation parcellaire.

L ‘importance de l’infestation est estiméc à partir d'observations sur la végétation adventice de parcelles mises en défens de traitements herbicides. Elle est quantitativement très variable, de 20 à 300 levées de plantules au $\mathrm{m}^{2}$, avec une médiane à 125. Les mauvaises herbes majeures et quelques caractéristiques de leur biotope sont indiquées. Les espèces les plus fréquentes et abondantes sont des annuelles : les vivaces sont généralement fréquentes mais peu abondantes. Enfin, la prospection systématique de cultures de mais a mis en évidence la présence de populations de Chenopodium album (sur le quart des parcelles visitées) et de Solamum nigrum résistantes à l'atrazine.
\end{abstract}

Mots clés additionnels : Mauraises herbes, biocínoses végétales, typologic des exploitations, résistance aux herbicides, Noyonnais.

Characteristics and dynamics of weeds in an area of field crops : the Noyonnais (Département de l'Oise, France).

After recalling the major importance of weed control and herbicide use in cultivated fields, the authors present an analysis of the efficiency and the effect of chemical weed control on the weed llora composition and emergence density in field crops in the Noyonnais. The estimation of weed population densities was done on plots without chemical control. The number of seedlings varied from $2010300 \mathrm{~m}$ ? with a median of 125 . The most common and frequent species were annual weeds : perennials were generally frequent but with a low density. For the main species, some environmental requirements were noted. In maize fields, systematic surveys showed the presence of triazine-resistant populations of Chenopodium album (in a quarter of the fields) and Solanum nigrum.

Additional key words: Weeds, vegetable biocenosis, typology of exploitations, herbicide resistance, Noyonnatis.

\section{INTRODUCTION}

Les régions de grande culture sont caractérisées par la dominance, dans l'assolement, des céréales et des cultures industrielles; lorsque l'élevage est présent, l'alimentation des animaux est assurée en grande partie par des fourrages annuels ou bisannuels. A ces cultures, implantées, travaillées et récoltées à l'aide de matériel performant, est associé un emploi généralisé de facteurs de production d'origine industrielle : engrais et pesticides, dont l'impact est prépondérant au niveau de la végétation adventice, et susceptible de modifier la situaltion malherbologique régionale.
Cette étude fait partie d'un programme multidisciplinaire de recherche sur l'environnement (P.I.R.E.N.) du C.N.R.S. : "Changement écologique et socioéconomique en région de grande culture ", animé par M. SebillotTe (1983). La région prospectée est celle du canton de GuisCarD, près de NOYON (60), qui présente les caractéristiques culturales déjà mentionnées. Des études récentes concernant la fertilité et les systèmes de production dans cette région sont en cours de publication.

Un des traits les plus caractéristiques de l'évolution des systemes de culture concerne le désherbage et l'uti- 

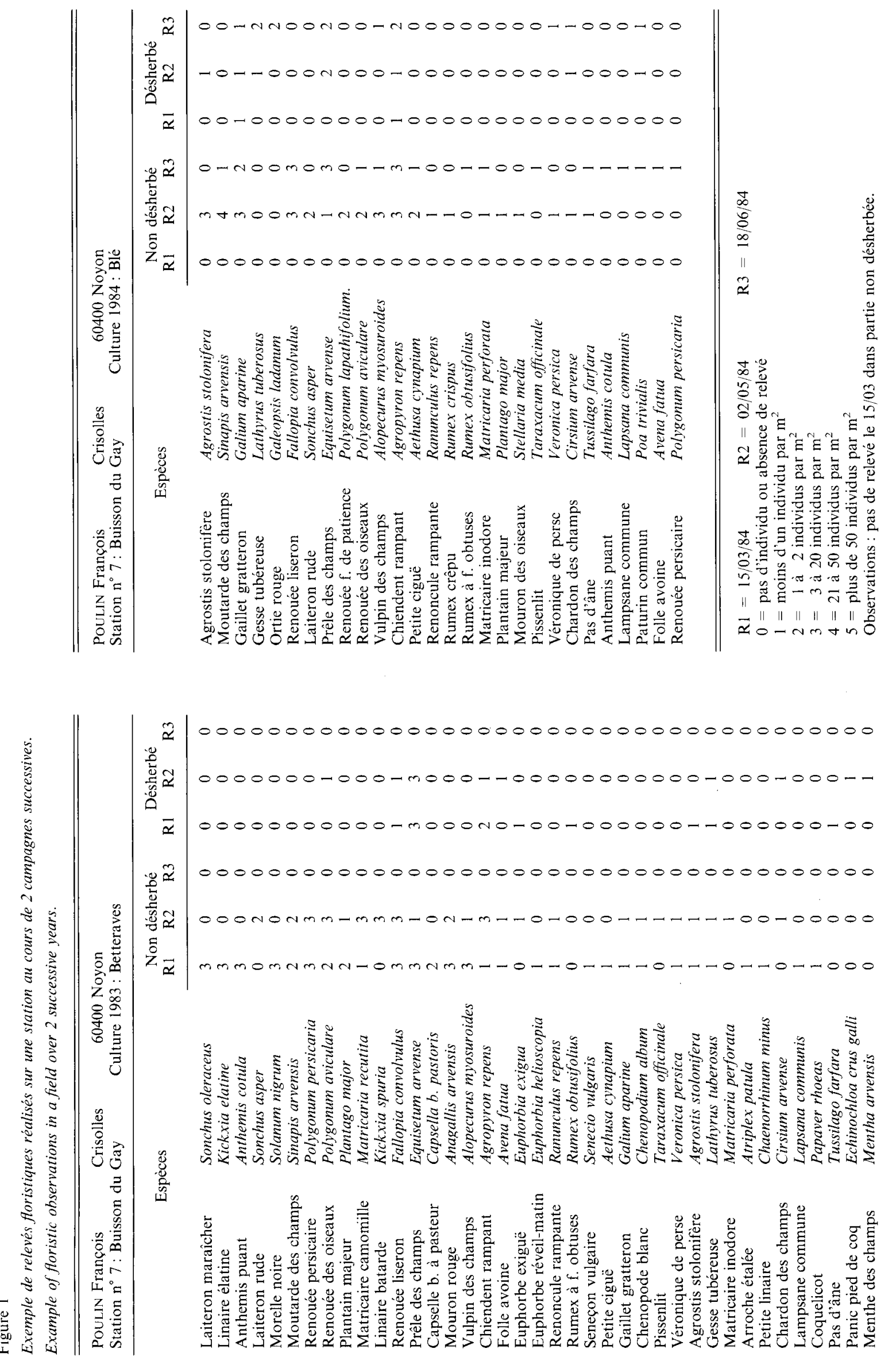
lisation des herbicides. Le présent travail tente de répondre aux questions: quelle est l'efficacité des moyens de lutte utilisés, comment le potentiel d'infestation en adventices est-il affecté, quels sont les problèmes qui se posent...?

\section{MÉTHODE}

La présence de mauvaises herbes dans les cultures peut être, en première approximation, considérée comme la conséquence des conditions pédo-climatiques dont les caractéristiques satisfont les exigences écologiques des espèces (BRAUN-BLANQUET, 1932; BOURNERIAS, 1984). L'abondance est une conséquence des techniques culturales mises en œuvre en ce lieu. La notion d'abondance fait référence à 2 variables :

- le potentiel d'infestation d'une parcelle qui est le nombre de diaspores (semences et bourgeons viables) contenus dans le sol. Il est lié aux itinéraires culturaux (techniques de préparation du sol, fertilisation, désherbage, etc...) et aux successions culturales;

- la densité d'infestation d'une culture qui est l'expression du potentiel d'infestation par levée des mauvaises herbes, compte tenu de la culture, des conditions culturales et climatiques de l'année.

Dans le présent travail, nous avons estimé la densité d'infestation d'un certain nombre de stations en absence de tout désherbage au cours d'une ou 2 années d'observation, ainsi que la densité d'infestation sur les stations homologues normalement désherbées. Une station est un emplacement homogène dans ses conditions physiques et biologiques sur lequel sont effectuées des observations. Elle occupe en moyenne une surface de $150 \mathrm{~m}^{2}$ dans une parcelle. Compte tenu de l'hétérogénéité pédologique, plusieurs stations par parcelles ont pu être étudiées. La connaissance de la densité d'infestation permet :

- d'apprécier à partir des relevés l'efficacité du désherbage tel qu'il est pratiqué normalement par l'agriculteur (comparaison des densités d'infestation des stations désherbées ou non),

- de relier les densités d'infestation d'une même station, sur cultures d'automne et de printemps, au passé cultural des parcelles dans un milieu pédoclimatique défini.

\section{A. Enquêtes phytotechniques}

Des enquêtes phytotechniques sont réalisées chez les agriculteurs. Elles ont essentiellement pour but de connaître la succession des cultures ainsi que l'intensité du désherbage chimique durant les 10 dernières années pour chaque parcelle étudiée. Elles renseignent également sur les caractéristiques de mise en place, de fertilisation et de désherbage de la culture, l'année où sont faites les observations floristiques.

\section{B. Etudes floristiques}

Dans l'impossibilité matérielle d'estimer directement le potentiel semencier et afin d'apprécier au mieux la diversité floristique par l'examen de la flore de surface, des observations sont effectuées sur chaque station mise en défens de désherbage pendant 2 années successives, l'une correspondant à une culture d'automne, l'autre à une culture de printemps (ou vice-versa). Les relevés de végétation sont réalisés sur la base d'une échelle de notation (BARRALIS, 1976) :

$$
\begin{aligned}
& 0=\text { Absence de l'espèce } \\
& 1=\text { Moins d' } 1 \text { individu par } \mathrm{m}^{2} \\
& 2=1 \text { à } 2 \text { individus par } \mathrm{m}^{2} \\
& 3=3 \text { à } 20 \text { individus par } \mathrm{m}^{2} \\
& 4=21 \text { à } 50 \text { individus par } \mathrm{m}^{2} \\
& 5=\text { Plus de } 50 \text { individus par } \mathrm{m}^{2}
\end{aligned}
$$

Ces estimations de densité sont effectuées 3 fois par an pour les cultures d'automne et 2 fois pour celles de printemps. Lors de l'exploitation des relevés on prend en compte les coefficients les plus élevés. La figure 1 montre un état édité des relevés floristiques concernant une station étudiée en 1983 et 1984 ; ils sont disponibles au laboratoire. Dans certains cas, la liste des espèces a été complétée par l'observation de levées de plantules en serre à partir de prélèvements de terre (4 $\mathrm{kg}$ environ) effectués dans chaque station.

\section{Stations étudiées}

L'inventaire quantitatif de la végétation est réalisé sur 47 stations réparties sur 44 parcelles appartenant à 21 exploitations agricoles. Leur répartition par communes, cultures, classes de texture de sol et types d'exploitation est donnée au tableau 1. Pour des raisons techniques, 20 stations seulement ont pu être étudiées sur 2 années successives suivant le protocole établi.

Concernant les successions culturales, nous n'avons pas observé de rotations typiques à part la rotation betteraves-blé. Cependant, il est possible de regrouper les stations en fonction de la fréquence des différentes cultures sur les 10 dernières années :

- betteraves-blé d'automne

8 stations

- betteraves-blé avec introduction d'escourgeon

9 stations

- betteraves-blé avec introduction de maïs

7 stations

- céréales avec pois ou haricots fréquents 6 stations

- céréales avec pommes de terre fréquentes

3 stations

- parcelle à passé fourrager marqué (dont prairie)

- non classable

5 stations

Total :

9 stations

47 stations

\section{Localisation de populations résistantes à l'atrazine}

Une prospection systématique des parcelles de maïs sur l'ensemble du canton de GuISCARD a été effectuée dans le but de mettre en évidence la présence chez certaines espèces de mauvaises herbes de populations résistantes à l'atrazine (GASQUEZ et al., 1982). En 1984, 60 parcelles ont été visitées; dans le cas d'infestations anormales sur cultures désherbées, des estimations de densité étaient réalisées, et des semences récoltées. Ces semences sont ensuite mises à germer au laboratoire de 
TABLEAU 1

Répartition des stations étudiées par communes, cultures, classes de texture du sol et types d'exploitations agricoles. Distribution of the fields studied by commune, crop, type of soil and cultivation system.

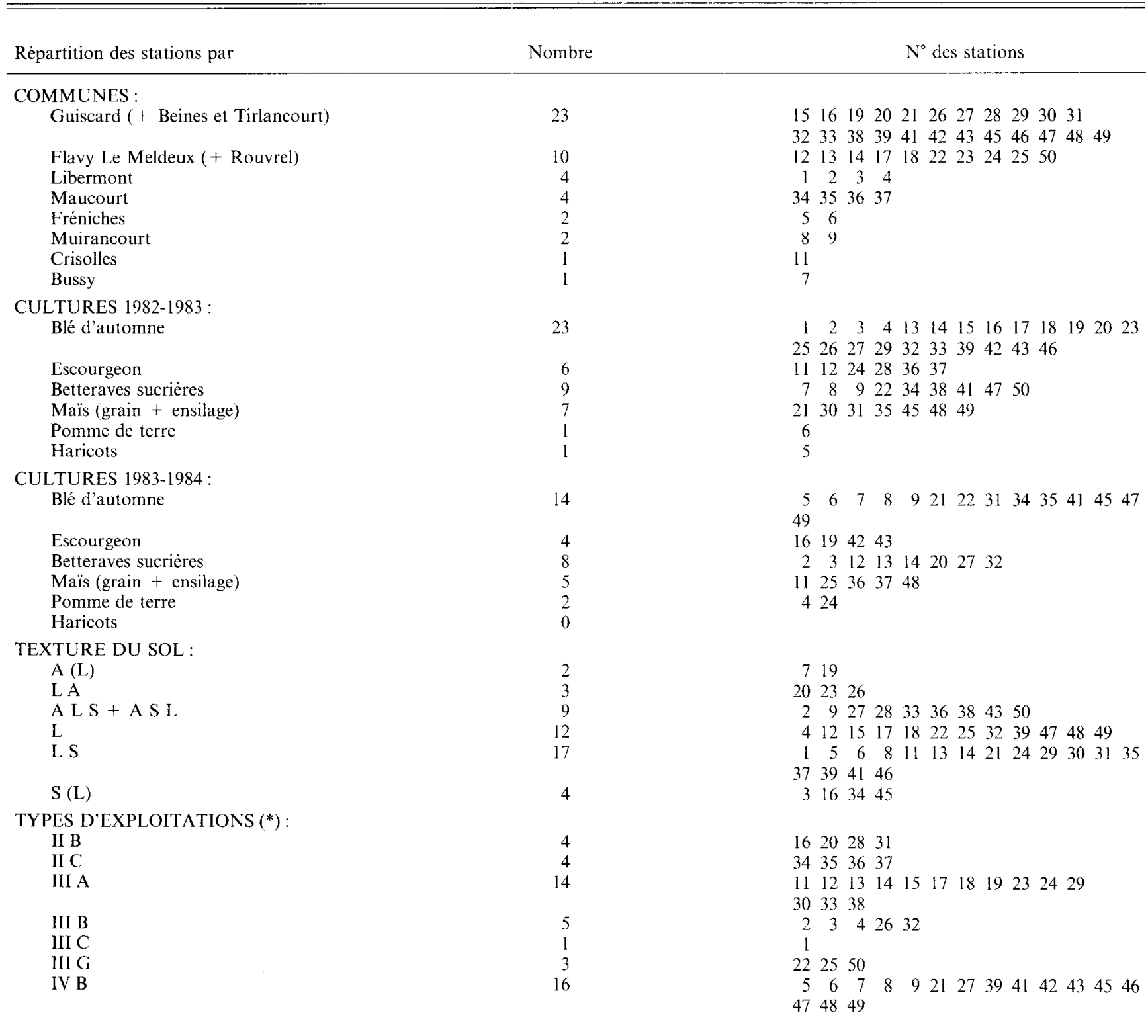

(*) Sources:

II B : Exploit. $<50$ ha, polyculture et polyélevage.

II C: Exploit. < 50 ha, élevage laitier intensif, cultures.

III A : Exploit. 50-80 ha, polyculture et polyélevage, 20-30 UGB.

III B : Exploit. 50-80 ha, grande culture et élevage intensif.

III C : Exploit. 50-80 ha, culture (blé-bett.) et lait intensifs.

III G : Exploit. 50-80 ha, grande culture, ateliers spécialisés.

IV B : Exploit. 100-150 ha, grande culture seule, 1 à 2 salariés.

façon à obtenir des plantules sur lesquelles sont pratiqués des tests de résistance à l'atrazine (DUCRUET \& GASQuez, 1978).

\section{RÉSULTATS}

\section{A. Etude quantitative de la végétation adventice}

Le nombre des espèces recensées au cours des 2 campagnes d'étude est de 119. Ces espèces sont banales et dénotent une pauvreté floristique liée entre autre à la relative homogénéité pédologique des stations étudiées : sols lessivés modaux très instables et battants, sols bruns lessivés stables peu battants et dans quelques stations à passé prairial récent, sols bruns faiblement lessivés sur sous sol argileux. Les classes de texture de sols sont présentées tableau 1.

Le tableau 2 regroupe les 44 espèces dont la fréquence (F p. 100) calculée à partir des 81 relevés effectués est supérieure à 10 p. 100 . L'abondance de ces espèces, évaluée suivant l'échelle à 6 indices, est présentée par classes de densité pour les 4 cultures les plus importan- 
TABLEAU 2

Fréquence ( $F$ p. 100$)$ des espèces calculée à partir de leur présence dans 81 relevés, ef fréquences dobservation calculées dans différentes cultures ( + ) avec leur répartition par classes de densité : I (moins de $\left.1 / \mathrm{m}^{2}\right), 2\left(1\right.$ à $\left.2 / \mathrm{m}^{2}\right), 3\left(3\right.$ à $\left.20 / \mathrm{m}^{2}\right), 4\left(21\right.$ à $\left.50 / \mathrm{m}^{2}\right)$ et $5\left(\mathrm{plus}\right.$ de $\left.50 / \mathrm{m}^{2}\right)$, pour les espèces dont F p. $l 00$ est supérieur à 10 .

Frequency $(F \%)$ of species calculated from 81 floristic observations, and frequency ( + ) calculated from different crops according to density classes:

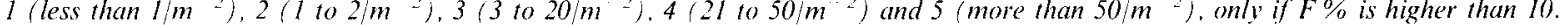

ESPĖCES

CLLTURES DOBSERVATION

\begin{tabular}{|c|c|c|c|c|c|c|c|c|c|c|c|c|c|c|c|c|c|c|c|c|c|c|c|c|c|c|}
\hline \multirow[b]{2}{*}{ Nom scientifique } & \multirow[b]{2}{*}{ Nom commun } & \multirow[b]{2}{*}{$\mathrm{F} \%$} & \multicolumn{6}{|c|}{ Blé } & \multicolumn{6}{|c|}{ Escourgeon } & \multicolumn{6}{|c|}{ Betteraves } & \multicolumn{6}{|c|}{ Maïs } \\
\hline & & & + & 1 & 2 & 3 & 4 & 5 & + & 1 & 2 & 3 & 4 & 5 & + & 1 & 2 & 3 & 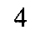 & 5 & + & 1 & 2 & 3 & 4 & \\
\hline & & & 97 & 21 & I1 & 24 & 11 & 30 & 99 & 0 & 11 & 44 & 33 & 11 & & 50 & & 31 & 0 & & 75 & & 17 & 5 & & \\
\hline Cirsilum arv & 1 & 16 & 79 & 71 & 8 & 0 & & 0 & 44 & 44 & 0 & 0 & 0 & 0 & 87 & 81 & 0 & 6 & 0 & 0 & 75 & 67 & 8 & 0 & 0 & \\
\hline Stellaria nedia & Mou & . & 71 & 26 & 8 & 34 & 3 & 3 & 77 & 0 & 11 & 44 & 11 & 11 & 56 & 44 & 0 & 6 & 6 & 0 & 50 & 8 & 17 & 8 & 17 & \\
\hline Matricaria perforata & Matri & 65 & 84 & 55 & 8 & 5 & 5 & 11 & 66 & 33 & 11 & 11 & 11 & 0 & 63 & 25 & 19 & 13 & 6 & 0 & 5 & 17 & 8 & 0 & O & \\
\hline Anagallis arvensis & Mou & & 53 & 45 & 3 & 5 & 0 & 0 & 78 & 67 & 11 & 0 & 0 & 0 & 82 & 44 & 13 & 25 & 0 & 0 & 66 & 25 & 33 & 8 & 0 & \\
\hline Capsella b & & & 59 & 40 & 13 & 3 & O & 3 & 78 & 56 & 22 & 0 & 0 & 0 & 81 & 50 & 25 & 0 & 6 & 0 & 58 & 33 & 25 & 0 & 0 & \\
\hline Galiur & & & 79 & 50 & 21 & 5 & 3 & 0 & 89 & 56 & 0 & 22 & 11 & 0 & 0 & 44 & 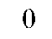 & 6 & 0 & 0 & & 17 & 0 & 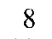 & & \\
\hline Chenot & & 2 & 40 & 32 & 5 & 3 & 0 & 0 & 22 & 11 & 11 & 0 & 0 & 0 & 100 & 44 & 25 & 19 & 13 & 0 & 100 & 33 & 17 & 33 & 17 & \\
\hline Matr & & 58 & 58 & 34 & 8 & 8 & & 5 & 33 & 11 & 11 & 11 & 0 & 0 & 5 & 38 & 0 & 25 & 6 & 6 & 60 & 17 & 8 & 17 & & \\
\hline Poly & & & 55 & 39 & 11 & 5 & & 0 & 55 & 33 & 11 & 11 & 0 & 0 & & 63 & 8 & 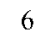 & 0 & - & & 33 & 0 & 8 & & \\
\hline Sinap & & 57 & 50 & 39 & 3 & 5 & & 0 & 66 & 44 & 11 & 11 & 0 & 0 & 9 & 44 & 19 & 0 & 6 & 0 & & 42 & 8 & 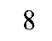 & & \\
\hline Poa ant & & 57 & 66 & 29 & 13 & 16 & 0 & 8 & 77 & 22 & 33 & 11 & 0 & 11 & 46 & 31 & 15 & 0 & 0 & 0 & 5 & 17 & 0 & 8 & & \\
\hline At'thu & & & 50 & 29 & 3 & 13 & 5 & 0 & 44 & 44 & 0 & 0 & 0 & 0 & & 50 & 0 & 19 & 0 & 0 & 2 & 25 & 17 & 0 & & \\
\hline Ver & & & 51 & 32 & 16 & 3 & & 0 & 77 & 11 & 22 & 33 & 0 & 11 & & 44 & 0 & 0 & 0 & 0 & & 33 & 0 & 8 & & \\
\hline Polygor & & & 37 & 34 & 0 & 0 & 0 & 3 & 44 & 44 & 0 & 0 & 0 & 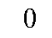 & & 31 & 6 & 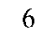 & 0 & 0 & 7 & 50 & ) & 17 & & \\
\hline Solan & & & 27 & 24 & 3 & 0 & & 0 & 11 & 11 & 0 & 0 & 0 & 0 & & 50 & 19 & 15 & 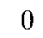 & . & 9 & 25 & 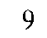 & -3 & & \\
\hline Euphon & & & 26 & 26 & 0 & 0 & & 0 & 33 & 33 & 0 & 0 & 0 & 0 & 3 & 50 & 13 & 0 & 0 & 0 & 33 & 33 & 0 & 0 & & \\
\hline Poa tr & & & 50 & 37 & 8 & 5 & & 0 & 55 & 44 & 0 & 11 & 0 & 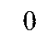 & & 13 & 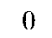 & 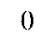 & 0 & . & 8 & 0 & 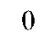 & 8 & & \\
\hline & & & 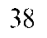 & 2 & 3 & 3 & & 0 & 33 & 22 & 11 & 0 & 0 & 0 & & & 0 & 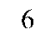 & 0 & & 1 & 25 & & 8 & & \\
\hline Ver & & & 51 & 32 & 8 & 8 & & 0 & 33 & 11 & 11 & 0 & 11 & 0 & & 13 & 0 & 0 & 0 & 0 & 0 & & 8 & 0 & & ) \\
\hline Mer & & & 37 & 34 & 0 & 3 & & 0 & 0 & 0 & 0 & 0 & 0 & 0 & & 31 & 6 & ( & 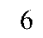 & 0 & & & & 7 & & \\
\hline & & & 35 & & 3 & 0 & 0 & 0 & 33 & 22 & 0 & 11 & 0 & & & & 0 & 0 & 0 & & 8 & 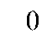 & 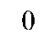 & & & \\
\hline Plan & & & 29 & 29 & 0 & 0 & & 0 & 33 & 33 & 0 & 0 & 0 & 0 & 5 & 19 & 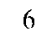 & 0 & 0 & 0 & 41 & 33 & 8 & 0 & & ) \\
\hline Viola & & & 18 & l & 0 & 0 & & 0 & 55 & 44 & 0 & 11 & 0 & 0 & & 19 & 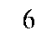 & . & . & 0 & 41 & 33 & & & & \\
\hline & & & 32 & & 0 & 0 & 0 & 0 & 22 & & 0 & & 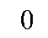 & & & & 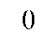 & & 0 & & 0 & & & & & \\
\hline & & & 13 & 13 & 0 & 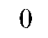 & 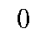 & 0 & 22 & 22 & 0 & 0 & 0 & 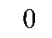 & 0 & 19 & 19 & 12 & 0 & 0 & 25 & 25 & 0 & 0 & & ) \\
\hline & & & 31 & 18 & 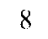 & 5 & & 0 & 33 & 22 & 0 & 11 & 0 & 0 & & 13 & 0 & a & a & 0 & 0 & 0 & 0 & 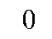 & & \\
\hline$A t r$ & & & 4 & 11 & 3 & 0 & 0 & 0 & 22 & 11 & 11 & 0 & 0 & 0 & & 1 & 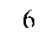 & 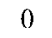 & 0 & 0 & 50 & 50 & & & & \\
\hline$R u$ & & & 23 & 18 & 5 & 0 & 0 & 0 & 33 & 33 & 0 & 0 & 0 & 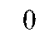 & & & 0 & 0 & 0 & 0 & 0 & 0 & $\theta$ & 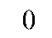 & & \\
\hline & & & 5 & 5 & 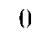 & 0 & & 0 & 11 & 11 & 0 & 0 & 0 & 0 & & 31 & 0 & 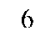 & 0 & 0 & 25 & 25 & 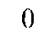 & 0 & & \\
\hline & & & 19 & 16 & 3 & 0 & & 0 & 44 & 44 & 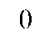 & 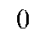 & o & & & & 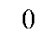 & 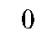 & 0 & & & & & & & \\
\hline & & 19 & 21 & 21 & () & 0 & 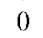 & 0 & 22 & 22 & 0 & 0 & 0 & 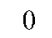 & 19 & 19 & a & 0 & 0 & 0 & 8 & 8 & 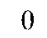 & 0 & & \\
\hline Sonch & & & 8 & 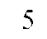 & 3 & 0 & 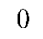 & 0 & 11 & 11 & 0 & 0 & 0 & 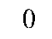 & & & 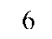 & 0 & 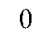 & 0 & 25 & 25 & 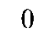 & & & \\
\hline Euphorb & & 16 & 8 & 8 & 0 & 0 & 0 & 0 & 11 & 11 & 0 & 0 & 0 & 0 & 37 & 31 & 6 & 0 & 0 & 0 & 16 & 8 & 8 & 0 & & 0 \\
\hline Chaen & & 16 & 5 & 5 & 0 & 0 & 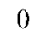 & 0 & 11 & 11 & 0 & 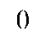 & 0 & 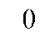 & 44 & 38 & 6 & 0 & 0 & 0 & 27 & 27 & 0 & & & \\
\hline Lam & & 14 & 16 & 13 & 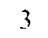 & 0 & 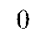 & 0 & 44 & 33 & 11 & & 0 & & 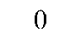 & & 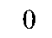 & 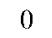 & 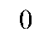 & 0 & 0 & 0 & 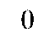 & & & \\
\hline onifera & & 13 & 10 & 5 & 0 & 5 & 0 & 0 & 11 & 0 & 11 & 10 & 0 & 0 & 19 & 19 & 0 & 0 & 0 & 0 & 17 & 17 & 0 & 0 & & 0 \\
\hline & & 13 & 27 & 24 & 3 & 0 & 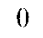 & 0 & 22 & 11 & 11 & 0 & 0 & 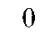 & 0 & 0 & 0 & 0 & 0 & 0 & 0 & 0 & 0 & 0 & & \\
\hline Filaginello & & 1. & 16 & 16 & 0 & 0 & 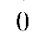 & 0 & 11 & 11 & 0 & & . & & 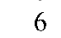 & & & 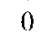 & 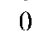 & 0 & 17 & 17 & 0 & 0 & & \\
\hline Spergula arvensis & Spergule des champs & 11 & 13 & 13 & 0 & 0 & 0 & 0 & 22 & 22 & 0 & 0 & 0 & 0 & 0 & 0 & () & 0 & 0 & 0 & 0 & 0 & 0 & 0 & 0 & \\
\hline
\end{tabular}

tes et statistiquement exploitables: 37 blés d'hiver, 17 betteraves, 13 maïs et 10 escourgeons ; ils représentent 95 p. 100 des relevés effectués. Cette liste est complétée par les espèces moins fréquentes suivantes: Anthemis cotula. Arenaria serpyllifolia, Artemisia vulgaris, Atriplex hastata, Calystegia sepium. Cerastium fontanum, Cerastium glomeratum. Cerastium semidecandrum, Chenopodium ficifolium, Chenopodium hybridum, Chenopodium polyspermum, Echinochloa crus galli, Filaginella uliginosa, Galeopsis ladanum, Galeopsis tetrahit, Geranium dissectum, Geranium pusillum, Geranium rotundifolium, Heracleum sphondylium, Holcus lanatus, Kickxia spuria, Lathyrus tuberosus, Lolium multiflorum, Mentha arvensis, Myosotis arvensis, Pastinaca sativa, Plantago lanceolata, Polygonum lapathifolium, Ranunculus repens, Rumex acetosa, Rumex crispus, Scleranthus annuus, Sonchus arvensis, Stachys annua, Stachys palustris, Stellaria graminea, Symphytum officinale, Taraxacum officinale, Tussilago farfara, Urtica urens et Viola arvensis.

\section{Interprétation globale}

La méthode d'étude nous permet d'estimer sur les 20 stations la densité moyenne des espèces levées, et d'en faire la somme : les coefficients de densité $1,2,3,4$ et 5 représentant respectivement 0,$5 ; 1,5 ; 11,5 ; 35,5$; et 75,5 individus par $\mathrm{m}^{2}$. L'histogramme de la figure 2 présente la fréquence des relevés dans les différentes classes d'infestation. Certaines parcelles sont assez peu infestées, d'autres beaucoup plus; l'amplitude du nombre de levées est voisin de 10 pour les classes extrêmes. 


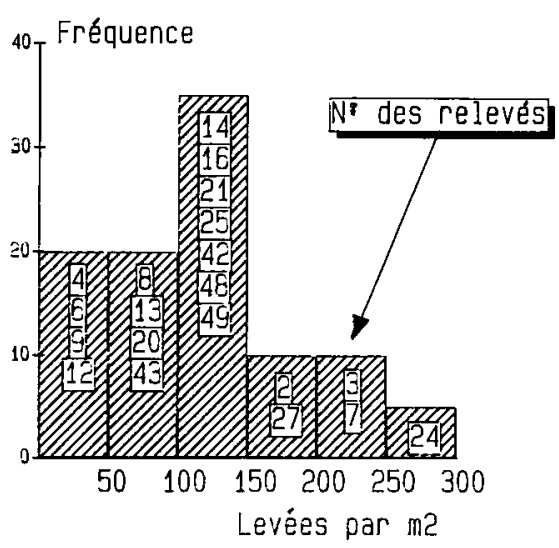

Figure 2

Répartition des 20 stations en fonction de leur densité d'infestation estimée par l'observation des levées au cours des 2 années successives comportant une culture d'automne et une culture de printemps (ou vice-versa)

Distribution of 20 fields according 10 weed flora abundance, as estimnted by seedling analysis over 2 successive vears with a winter crop and a spring (rop (or vice versa).

\section{Interprétation par espèces}

a) Espèces fréquentes (F. p. 100 supérieur à 75)

- dans toutes les cultures

- avec une densité élevée en cultures d'automne: Alopecurus myosurö̈des.

- avec une densité moyenne en cultures de printemps : Anagallis arvensis.

- avec une densité faible: Agropyron repens, Capsella bursa-pastoris, Cirsium arvense.

- uniquement en cultures d'automne, avec une densité élevée: Galium aparine, Poa annua.

- uniquement en cultures de printemps, avec une densité élevée: Chenopodium album, Solunum nigrum.

b) Espèces moyennement fréquentes (F. p. 100 compris généralement entre 75 et 30 )

- dans toutes les cultures

- avec une densité plus élevée en cultures d'automne: Stellaria media, Veronica persica.

- avec une densité plus élevée en cultures de printemps : Mercurialis annua, Polvgonum persicaria.

- avec des densités moyennes: Matricaria perforata, M. recutita, Fallopia convolvulus, Polygonum ariculare, Sinapis arvensis, Aethusa cynapium.

- avec des densités faibles: Euphorbia helioscopia, Papaver rhoeas, Equiselum arvense, Plantago major.

— uniquement en cultures d'automne

- avec une densité plus élevée en cultures dautomne: Veronica hederifolia, Apera spica venti.

- avec une densité faible: Poa trivialis. c) Espèces peu fréquentes (F.p. 100 généralement inférieur à 30 ) et en faibles densités

- dans toutes les cultures: Agrostis stolonifera, Avena fatua, Filaginella uliginosa, Juncus bufonius, Rumex obtusifolius, Viola tricolor.

- plus fréquentes ou abondantes en cultures d'automne: Aphanes arvensis, Fumaria officinalis, Lamium purpureum, Lapsana communis, Spergula arvensis.

- plus fréquentes et abondantes en cultures de printemps: Atriplex patula, Chaenorrhinum minus, Euphorbia exigua, Kickxia elatine, Sonchus asper, Sonchus oleraceus.

\section{B. Etude des relations entre la densité des principales mauvaises herbes et les facteurs stationnels}

A partir de 53 relevés pour lesquels nous avons pu collecter suffisamment de renseignements phytotechniques, nous avons pratiqué une analyse en composantes principales (ACP) sur 13 variables floristiques (espèces dont la fréquence est supérieure à $50 \mathrm{p} .100$ pour ce groupe de relevés) et 38 variables stationnelles polytomiques, ordonnées ou entières. Il s'agit de :

- la texture, la structure et le pH du sol,

- la fréquence de différentes cultures dans la succession culturale,

- Ia fertilisation azotée, phosphatée et potassique,

- l'efficacité du désherbage par comparaison des parcelles traitées et non traitées.

- l'intensité du désherbage chimique estimé sur ces 10 dernières années compte tenu des produits utilisés, du nombre des traitements et éventuellement des doses utilisées,

- la surface de l'exploitation et l'âge de l'agriculteur.

Les 5 premières composantes absorbent respectivement : 13,$4 ; 10,0 ; 8,0 ; 7,2$ et 6,9 p. 100 de la variabilité globale, mais seules les 3 premières manifestent pour l'ensemble des variables une corrélation moyenne significative. Nous avons représenté figure 3 les 53 relevés dans le premier plan factoriel et figure 4 les 38 axes unitaires relatifs à ce même plan.

On observe figure 4 que la première composante est liée négativement à l'intensification, caractérisée par une forte pression de désherbage ces dernières années et par une très bonne efficacité de celui-ci l'année des observations. La fertilisation potassique y est importante. Blé et betteraves reviennent fréquemment dans la succession alors que l'escourgeon et les cultures fourragères y sont rares puisque liés positivement à l'axe 1. Enfin, ces parcelles appartiennent à des exploitations assez importantes cultivées par des agriculteurs plutôt jeunes.

La deuxième composante est liée essentiellement à la fertilisation azotée et phosphatée apportée sur des parcelles au sol argileux et argilo-limoneux de $\mathrm{pH}$ élevé. L'escourgeon revient fréquemment dans les rotations. Les parcelles sur sols limoneux battants sont issues d'anciennes pâtures et reçoivent peu de fertilisants minéraux. 


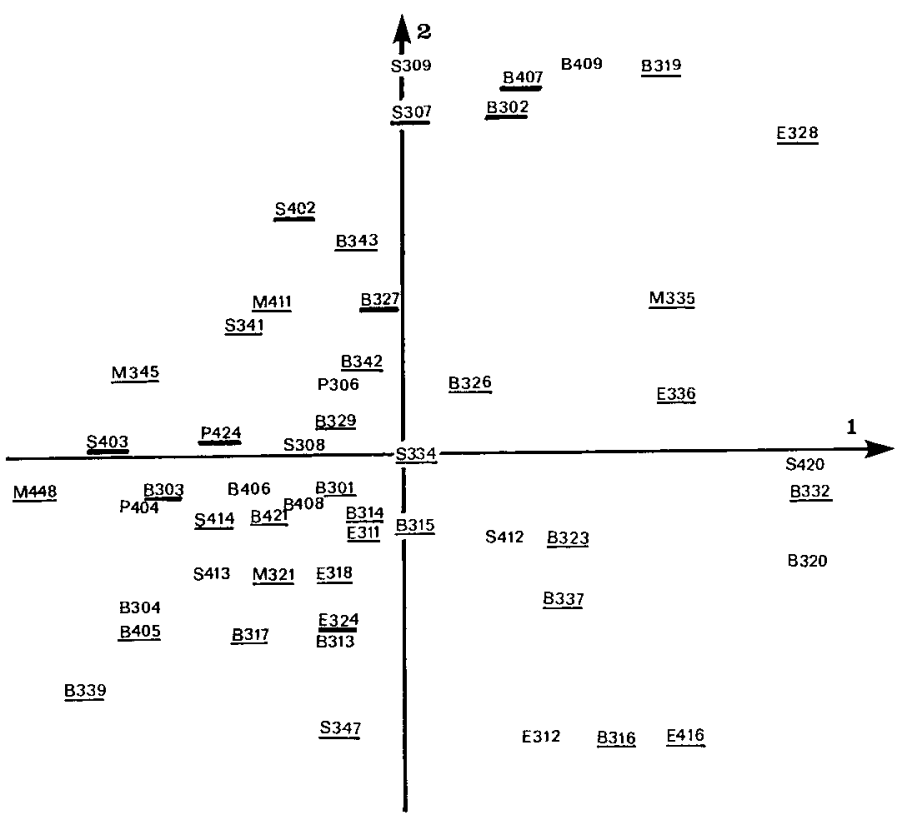

Figure 3

Analyse en composantes principales sur $53^{\circ}$ relevés et 38 variables Représentation des stations dans le plan des composantes 1 et 2.

Code des stations: $1 \quad 2 \quad 3 \quad 4$.

$I=$ culture $: B=$ blé, $E=$ escourgeon, $S=$ betterave sucrière, $M=$ maïs, $P=$ pomme de terre.

$2=$ année d'observation : $3=1983,4=1984$

$3+4=$ numéro de la station.

Codes non soulignés $=$ Relevés sur parcelles d'infestation inférieure $\dot{a}$ 100 plantes par $\mathrm{m}^{2}$.

Codes soulignés $-=$ Relevés sur parcelles d'infestation comprise entre 100 et 150 plantes par $\mathrm{m}^{2}$.

Codes soulignés $\underline{=}=$ Relevés sur parcelles d'infestation supérieure à 150 plantes par $\mathrm{m}^{2}$

Principal components analysis with 53 floristic observations and 38 variables. Representation of the stations on the first two axes.

Code of the stations: $1 \quad 2 \quad 3 \quad 4$.

$I=$ crop : $B=$ winter wheat, $E=$ winter barley, $S=$ sugar beet, $M=$ maize, $P=$ potato.

$2=$ year of observation: $3=1983,4=1984$

$3+4=$ station number.

Codes not underlined $=$ Field infestations lower than 100 seedlings per $m^{2}$

Codes underlined with $-=$ Field infestation between 100 and 150 seedlings per $m^{2}$.

Codes underlined with $-=$ Field infestation higher than 150 seedlings per $m^{2}$.

Les coefficients de corrélation entre les composantes principales et les variables floristiques permettent de caractériser l'environnement favorisant les fortes ou faibles infestations des principales mauvaises herbes.

- Matricaires (Matricaria perforata, M. recutita): localisés préférentiellement en sols limoneux sur parcelles à successions blé-betteraves, désherbées de façon intensive, généralement sur des exploitations de 100150 ha (surface maximale des exploitations étudiées).

- Gaillet (Galium aparine) : trouvé essentiellement sur sol argilo-limoneux, en successions où l'escourgeon est fréquent ou (et) sur parcelles antérieurement en prairies; l'intensité du désherbage y est faible.

- Mouron des oiseaux (Stellaria media) et pâturin annuel (Poa annua) : présents en sols limoneux à $\mathrm{pH}$ bas, sur parcelles encore récemment pâturées ; exploitations de faibles tailles.

- Chiendent rampant (Agropyron repens) : trouvé essentiellement en sols argilo-limoneux, de $\mathrm{pH}$ élevé, sur

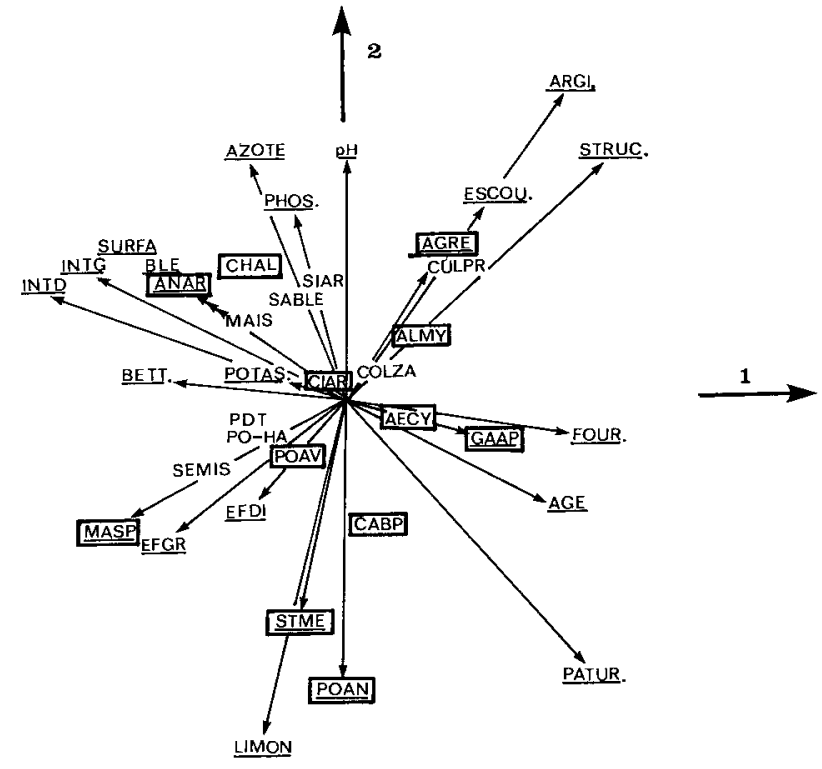

Figure 4

Analyse en composantes principales sur 53 relevés et 38 variables Représentation des axes unitaires.

Les variables soulignées ont un coefficient de corrélation significatif avec au moins 1 des axes.

Variables espèces: $A E C Y=$ Aethusa cynapium, $A G R E=$ Agropyron repens, $A L M Y=$ Alopecurus myosuroïdes, $A N A R=$ Anagallis arvensis, $C A B P=$ Capsella bursa-pastoris, $C H A L=$ Chenopodium album, $C I A R=$ Cirsium arvense, $G A A P=$ Galium aparine, $M A S P=$ Matricaria sp., $P O A N=$ Poa annua, $P O A V=$ Polygonum aviculare, $S I A R=$ Sinapis arvensis, $S T M E=$ Stellaria media.

Variables stationnelles: $A R G I .=$ taux d'argile, $L I M O N=$ taux de limons, $S A B L E=$ taux de sables, STRUC. = structure du sol, $p H=$ $p H, S U R F A .=$ surface des exploitations, $A G E=$ age des exploitants, SEMIS $=$ date de semis, AZOTE = fertilisation azotée, PHOS. $=$ fertilisation phosphatée, EFDI = efficacité herbicide anti-dicotylédones, $E F G R=$ efficacité herbicide anti-graminées, $I N T D=$ intensité $d u$ désherbage anti-dicotylédones, INTG = intensité du désherbage antigraminées

Variables culturales; Importance dans la rotation du blé $=B L E$, des betteraves sucrières $=B E T T$. de l'escourgeon $=E S C O U$. du maïs $=$ MAIS, des pois-haricots $=P O-H A$, du colza $=C O L Z A$, des pommes de terre $=P D T$, de la prairie $=P A T U R$, des cultures fourragères $=$ $F O U R$., des cultures de printemps $=C U L P R$

Principal component analysis with 53 floristic observations and $38 \mathrm{va}$ riables. Unitary axes.

Underlined variables have a significant correlation coefficient with at least I of the principal axes.

Species variables: $\quad A E C Y=$ Aethusa cynapium, $A G R E=$ Agropyron repens, $A L M Y=$ Alopecurus myosuroides, $A N A R=$ Anagallis arvensis, $C A B P=$ Capsella bursa-pastoris, $C H A L$ Chenopodium album, $C I A R=$ Cirsium arvense, $G A A P=$ Galium aparine, $M A S P=$ Matricaria sp., $P O A N=$ Poa annua, $P O A V=$ Polygonum aviculare, $S I A R=$ Sinapis arvensis, $S T M E=$ Stellaria media.

Station variables: $A R G I .=$ clay content, $L I M O N=$ silt content, $S A B L E=$ sand content, STRUCT. = structure, $p H=p H$, SURFA. = farm size, $A G E=$ age of farmer,$S E M I S=$ seedling date, AZOTE $=$ nitrate fertilizer, PHOS. $=$ phosphate fertilizer, EFDI = antidicotyledonous herbicide efficacy, EFGR = antigramineous herbicide efficacy, INTD = antidicotydelonons control, INTG = antigramineous control.

Crop variables: importance of wheat $=B L E$, sugar beet $=B E T T$. winter barley $=E S C O U$. maize $=M A I S$, peas-beans $=P O-H A$, rapeseed $=C O L Z A$, potatoes $=P D T$, grassland $=$ PATUR., fodder crops $=F O U R$. and spring crops $=C U L P R$. in the rotation.

parcelles où l'escourgeon revient fréquemment; exploitations de taille importante.

- Petite ciguë (Aethusa cynapium) et vulpin des champs (Alopecurus myosurö̈des): on trouve ces espèces en fortes densités sur parcelles où domine le colza dans la succession. 
- Moutarde (Sinapis arvensis), chardon des champs (Cirsium arvense) et renouée des oiseaux (Polygonum aviculare): présents sur sols bien structurés dans des parcelles où le maïs revient fréquemment.

- Chénopode blanc (Chenopodium album) : abondant sur successions à maïs fréquent dans lesquelles l'intensité du désherbage est faible.

La confrontation de la figure 3 et de la figure 2 montre clairement qu'aucune des variables utilisées en ACP (y compris l'intensité du désherbage depuis 10 ans) n'explique globalement l'infestation plus ou moins importante des parcelles. En effet, les stations $n^{\text {os }} 3,4,6,8$ et 24 par exemple, caractérisées par une intensification importante des facteurs de production (fertilisation importante, forte pression de désherbage...) restent très proches les unes des autres dans le plan des composantes 1 et 2 de la figure 3, alors que l'importance des levées observées (fig. 2) est très différente.

\section{Populations résistantes aux triazines}

La carte de la figure 5 présente la distribution des parcelles dans lesquelles on a observé des individus résistants. Sur 60 parcelles, 3 renferment des morelles (Solanum nigrum L.) et 14 des chénopodes blanes (Chenopodium album L.) résistants aux herbicides de la famille des triazines. Près du quart des parcelles de mais sont donc infestées en chénopodes résistants; cette valeur est peut être sous-estimée car des parcelles ont pu être traitées par un herbicide de post-levée, masquant alors la présence d'individus résistants. La densité de chénopodes dans certaines parcelles est telle que toute récolte est impossible.

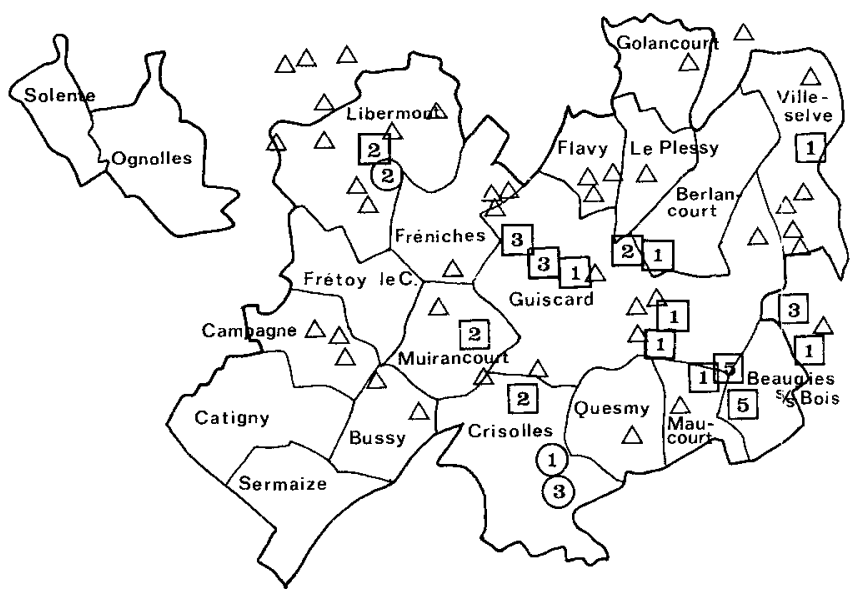

Figure 5

Répartition des populations de Chenopodium album et de Solanum nigrum résistantes aux triazines.

$\triangle$ Maïs sans plante résistante.

$\square$ Maïs avec chénopodes résistants lles chiffres reportent à l'échelie de densité).

Maïs avec morelles résistantes.

Distribution of atrazine-resistant populations of Chenopodium album and Solanum nigrum.

$\triangle$ Maize without resistant plants.

Maize with resistant $\mathrm{C}$. album.

Maize with resistant $\mathrm{S}$. nigrum.
La résistance des chénopodes s'est installée principalement sur les communes possédant des exploitations où l'assolement en maïs (ensilage surtout) est important. Les 3 populations de morelles résistantes ont été observées dans des communes où la part de l'assolement en betteraves est plus grande. Il faut noter enfin, qu'à travers les enquêtes phytotechniques les cultures de maïs ne paraissent pas avoir fait l'objet d'un désherbage intensif, ce qui suggère qu'un tel désherbage ne se justifiait pas et que l'apparition de plantes résistantes est vraisemblablement récente.

\section{DISCUSSION}

\section{A. Evolution récente de la flore adventice dans le Bassin Parisien et le nord de la France}

Une image de la flore adventice du département de l'Oise est donnée dès le milieu du XIX $^{\mathrm{e}}$ siècle par H. RoDIN (1863) et, suivant l'auteur elle est un bon marqueur de l'évolution de l'agriculture et de ses techniques: «Leur statistique (des adventices) fournit une donnée utile au point de vue de l'étude de la végétation spontanée en même temps qu'elle indique l'état de l'agriculture dans un pays... leur existence est entièrement liée aux progrès de l'agriculture ".

En comparant la fréquence de certaines espèces dans les flores et catalogues de cette époque (DE VICQ, 1883 ; Graves, 1857) aux listes actuellement établies (BARRALIS, 1980), on constate la diminution ou la disparition de très nombreuses espèces (oligotrophes, calcifuges, psammophiles, ...) telles: Agrostemma githago, Centaurea cyanus, Lithospermum arvense, Ranunculus sardous, Sherardia arvensis, ... et l'apparition ou la prolifération d'autres: Amaranthus retroflexus, Chaenorrhinum minus, Lamium amplexicaule, Matricaria perforata, $M$. recutita, Viola arvensis, Solanum nigrum, Veronica persica, ...

La banalisation de la flore adventice est la conséquence de la conjonction de causes de régression de certaines espèces (AYMONIN, 1973), et de l'envahissement par les espèces nitrophiles favorisées par les techniques mises en cuvre dans les systèmes de culture actuellement les plus répandus. Dans le département de l'Oise, en 1860 (RodIN, 1863), la surface cultivée en betteraves sucrières représentait 0,5 p. 100 de la S.A.U.; elle atteignait 16 p. 100 en 1980. Le maïs, autrefois inexistant représentait cette même année 10,6 p. 100 de la S.A.U.. L'assolement a donc considérablement évolué et le cortège des adventices pour les 2 cultures citées se limite à la prolifération des renouées, chénopodes, mercuriales et liserons.

Les observations réalisées sur le canton de Guiscard illustrent les constatations générales que nous venons d'évoquer, exception faite pour Amaranthus retroflexus. Cette espèce, observée dans une seule station hors échantillonnage, ne s'est pas répandue comme on aurait pu s'y attendre avec la culture de la betterave et l'extension des zones cultivées en maïs. Avena fatua, espece ignorée par RoDIN en 1863 et mentionnée comme «assez commune » par DE VICQ en 1883, dont on pouvait craindre l'extension, a eu un développement 
très limité (tabl. 2). Sur les stations étudiées, la dominance des limons et limons sableux confère au sol une compacité en milieu humide peu favorable au développement de ces 2 espèces.

L'importance des mauvaises herbes vivaces est aussi caractéristique de l'évolution des techniques culturales, des soins d'entretien et de la lutte chimique. Le département de l'Oise et le canton de Guiscard n'échappent pas à cette règle : le chiendent, les chardons et les prêles, encore actuellement difficiles à détruire par voie chimique, sont fréquemment favorisés, multipliés et dispersés par les façons culturales.

\section{B. Recherche des causes de prolifération de certaines adventices}

Les infestations parcellaires (en l'absence de désherbage) traduisent une forte densité en semences dans les sols alors que ceux-ci ont été désherbés mécaniquement de tout temps et régulièrement avec les herbicides depuis plus de 30 ans. On peut tenter de quantifier le potentiel d'infestation en semences si on considère, en moyenne. que 5 à 10 p. 100 des semences viables présentes dans le sol donnent une plantule (BARRALIS, 1979 ; BARRALIS \& Chadoeuf, 1980; Roberts, 1981). Les valeurs des classes d'infestation de la figure 2 sont alors à multiplier par 10 à 20. L'effectif de la classe modale se situe entre 1000 et 3000 semences par $\mathrm{m}^{2}$. A titre indicatif, de récentes estimations ont montré que les valeurs médianes du potentiel semencier se situaient de 4360 semences par $\mathrm{m}^{2}$ en Grande-Bretagne (ROBERTS \& CHANCELLOR, 1986) à 5100 semences par $\mathrm{m}^{2}$ en France (BARRAlis \& Chadoeuf, 1987).

La comparaison entre les parcelles traitées ou non montre que le désherbage des cultures pour les campagnes 82-83 et 83-84 a été efficace sur dicotylédones annuelles et satisfaisant sur graminées annuelles, sauf pour le vulpin des champs dans quelques parcelles (principalement en 1983).

Le désherbage limite donc l'infestation en mauvaises herbes mais ne permet pas l'élimination de tous les porte-graines qui assurent ainsi le réapprovisionnement du sol. Sachant que, selon les espèces, les semences peuvent se conserver plus ou moins longtemps dans le sol (CHAdoeuf et al., 1984), le potentiel d'infestation est maintenu, nécessitant au fil des successions culturales un désherbage systématique.

L'infestation actuelle par les vivaces est limitec. Chiendent et chardon sont fréquents mais rarement abondants et les prêles, présentes en situation de sols très humides et (ou) compactés, ont une densité qui dépasse rarement 2 plantes feuillées au $\mathrm{m}^{2}$.

Cependant, la notion de potentiel d'infestation que nous avons développée dans la méthodologie, s'applique mal aux espèces vivaces. Les unités de multiplication et de dispersion sont des semences (chardon des champs, tussilage, pissenlit, rumex, ...), mais aussi des rhizomes et drageons (chiendent rampant, agrostide stolonifère, prêle, chardon, tussilage) ou des tubercules (gesse). S'il est facile à l'aide d'un herbicide classique de détruire les plantes issues de semis, il est beaucoup plus difficile de lutter contre les plantes établies.

L'originalité des espèces possédant des rhizomes réside dans leur capacité à régénérer un individu par fragment. Toute technique culturale favorisant cette fragmentation assure donc la multiplication de l'espèce. Sur le territoire étudié. les infestations de chiendent semblent être assez bien contenues; pour les limiter, il suffirait de réduire les interventions de déchaumage utilisant des disques, de ramener et laisser sécher en surface les rhizomes ou d'envisager un traitement chimique. Si le chiendent, qui prolifère en terres lourdes, n'est pas à son optimum écologique, les prêles par contre se développent très bien dans les argiles et limons sableux, et il n'est pas souhaitable d'utiliser la méthode générale de lutte décrite pour le chiendent (rhizomes trop profonds et cassants, absence de lutte chimique efficace); elles risquent de poser des problèmes par la suite.

\section{CONCLUSION}

Les mauvaises herbes des cultures peuvent servir de marqueur de l'évolution des techniques culturales dans la mesure où il est possible de corréler la présence et labondance de certaines espèces à des pratiques culturales définies. Pour les espèces annuelles, il nous a été permis de préciser certaines de ces relations, mais pour les vivaces, en l'absence de point de repère antérieur, une telle analyse n'a pas été possible.

L'analyse parcellaire réalisée au cours de 2 campagnes agricoles, nous montre une bonne efficacité immédiate. mais une moindre efficacité à long terme du désherbage chimique. En effet, il se maintient dans le sol un potenticl d'infestation toujours élevé, tant pour les espèces que l'on sait être moins sensibles aux traitements herbicides (la petite cigüe et les véroniques par exemple). que pour l'ensemble des autres, globalement mieux maîtrisées.

Il ne faut pas oublier aussi que dans un système assolé se succèdent des cultures et des traitements spécifiques à ces cultures, dont laction ne se manifeste annuellement que sur une fraction (à peine 10 p. 100 en moyenne) de la flore adventice: en particulier celle qui peut lever dans les conditions d'environnement qui suivent le semis des cultures de printemps. La survie des semences enfouies, l'efficacité variable d'une année à l'autre mais jamais totale du désherbage, les rotations et leurs conséquences sur la levée des adventices, sont autant de facteurs qui ralentissent l'évolution de la flore.

Le fait qu'en l'absence de désherbage une seule année. et malgre une action qui s'exerce quasi systematiquement depuis plus de 30 ans, on puisse observer des communautés souvent supérieures à 100 individus par $\mathrm{m}^{2}$, laisse penser qu'il est difficile d'atteindre par le désherbage chimique un seuil tel que ce désherbage devienne totalement inutile. Dans ces conditions, puisqu'il faut annuellement désherber. on peut se demander s'il faut vraiment tenter systématiquement de tout détruire, sachant qu'il est utopique de croire que l'on puisse éliminer toutes les mauvaises herbes. N'est-il pas préférable de maintenir, à moindre frais, la végétation adventice en deçà d'une limite définie en fonction de la nature des mauvaises herbes et de la culture dans laquelle elles se développent?

Toutefois il ne s'agit pas de se cantonner à une routine de désherbage; la sélection de populations résistantes que nous avons évoquée avec le chénopode et la morel- 
le, oblige les agriculteurs à tenir compte de la réaction de la flore. Des problèmes nouveaux se posent, ils peuvent être techniquement résolus mais engendrent, sinon une contrainte au niveau des rotations, au moins une augmentation du coût du désherbage.

Recule 9 mai 1988 Accepté le 9 juillet 1988

\section{REMERCIEMENTS}

Les auteurs remercient chaleureusement leurs collègues du Laboratoire de Malherbologie: Messieurs R. Chadoeur, P. GiRARD pour lcur collaboration aux analyses floristiques, J. GASQUEZ pour la reconnaissance des populations atrazine-résistantes, ainsi que l'équipe de la Chaire d'Agronomie et du S.A.D., I.N.A. Paris-Grignon animée par M. Sebillot Te, particulièrement L. ServetTAz.

\section{RÉFÉRENCES BIBLIOGRAPHIQUES}

Aymonin G. G., 1973. Observations sur le processus de régression des adventices des cultures ( Mauvaises herbes») et conséquences biocéoénologiques. VI Colloque Int, Ecol. Biol. Mauraises Herbes, Marseille, 105-115.

Barralis G., 1976. Méthode d'étude des groupements adventices des cultures annuclles : Application à la Côte-d'Or. $V^{e}$ Colloque Int. Ecol. Biol. Mauvaise's Herbes, Dijon, 59-68.

Barralis G., 1979. Bases écologiques de la lutte contre les mauvaises herbes dans les cultures annuelles. Colloque CNRS Ecologic' 't Léleloppement, 283-291.

Barralis G., 1980. La répartition des mauvaises herbes. Perspectives Agricoles, 42, 10-12

Barralis G., Chadoeuf R., 1980. Etude de la dynamique d'une communauté adventice. l. Evolution de la flore adventice au cour du cycle végétatif d'une culture. We'd Res., 20, 231-237.

Barralis G., Chadoeuf R., 1987. Potentiel semencier des terres arables. Weed Research, 27, 417-424.

Bournerias M., 1984. Guide des groupements végétaux de la Région Parisienne. Masson. SEDES, Paris, 496 p. 30 édition.

Braun-Blanquet J., 1932-1962. Plant sociologt. The study of plant communities. Hafner Publishing Company, $439 \mathrm{p}$.
Chadoeuf R., Barralis G., Lonchamp J.-P., 1984 . Evolution du potentiel semencier de mauvaises herbes annuelles dans un sol cultivé VII Colloque Inter. Ecol. Biol. Sist. Mawvaises herbes, Paris, 29-35.

Ducruet J. M., Gasquez J., 1978. - Observation de la fiuorescence sur feuilles entières et mise en évidence de la résistance chloroplastique à l'atrazine chez Chenopodium album L.. Chemosphere, 8, 691-696.

Gasquez J., Barralis G., Aigle N., 1982. Distribution et extension de la résistance chloroplastique aux triazines chez les adventices annuelles en France. Agronomie, 2, 119-124

Graves L., 1857. Catalogue des plantes observées dans l'étendue du département de l'Oise. Beauvais.

Roberts H. A., 1981. Seed banks in soil. Advances in Applied Biology, 6. 1-55.

Roberts H. A., Chancellor R. J., 1986. Seed banks of some arable soils in the English Midlands. Weed Research, 26, 251-257.

Rodin H., 1863. Esquisse de la végétation du département de l'Oise Bull. Soc. Acad. Archéol. Sc. ét Art. du Dépt. de l'Oise, 354-507.

Sebillotte M., 1983. Changement écologique et socio-économique en région de Grande Culture. Colloque "Recherches sur l'enviromement rural, Bilan et Prospectives ", Paris, $41 \mathrm{p}$

de Vicq E., 1983. Flore du departement de la Somme. Abbeville. 